\title{
SIMULATING DRUG-TARGET INTERACTION USING LARGE-SCALE MOLECULAR DYNAMICS AND FUZZY-ART
}

\author{
ANKUSH RAI*, JAGADEESH KANNAN R \\ School of Computing Science \& Engineering, VIT University, Chennai, Tamil Nadu, India. Email: ankushressci@gmail.com
}

Received: 28 December 2016 Revised and Accepted: 10 May 2017

ABSTRACT

Introduction: The examination of bio-molecular associations between a complex drug compound and its target is of foremost significance for the improvement of new biomarkers or bio-responsive compounds.

Method: In this study, we exhibited a combinatorial technique of simulation of molecular dynamics (MD) and fuzzy-ART to focus on the coupling factors of the molecular binding process and its intermediary transitioning state. Here, MD simulations divided into microsecond length enable us to watch an inter-molecular coupling event, taking after different dynamical pathways and accomplishing ordered binding assembly of molecules.

Results and Discussion: Results from such simulations are used to evaluate parameters corresponding to its thermodynamic and molecular kinetic properties, getting a decent concurrence with accessible experimental information. Utilizing machine learning algorithms in conjunction with MD simulations could enhance the productivity for identifying key parts of drug-target binding and localization.

Keywords: Fuzzy-ARTMAP, Drug-target interaction, Biomolecular interaction

(C) 2017 The Authors. Published by Innovare Academic Sciences Pvt Ltd. This is an open access article under the CCBY license (http://creativecommons org/licenses/by/4. 0/) DOI: http://dx.doi.org/10.22159/ajpcr.2017.v10s1.19973

\section{INTRODUCTION}

The study on coupling mechanism of D Protein-ligand is the means of recognizing therapeutic effect of medications, and understanding of this procedure is of first centrality in medication revelation [1]. Revealing key data points in atomistic interaction of protein-ligand for assessing binding configuration will help in effectively designing bio-responsive compounds, while foreseeing the free energy and molecular dynamics (MD) associated with this procedure [2]. In this regard, MD has been comprehensively adopted [3]. Nowadays, it is possible to extensively reproduce frameworks of a couple of hundred thousand atoms, portraying various unthinking parts of the protein-ligand process to achieve a stable binding configuration. In a few previous cases, computational techniques using massive parallel models gave a full dynamical portrayal of proteinligand, bearing estimations of free energy and molecular kinetics associated with the procedure, with various degrees of exactness [4-8].

Transition-state analogs (TSAs) are artificially engineered structures that take after enzymatic adjusting states similarly as geometric and electrostatic components. TSA ligands are among the most-fit enzymatic inhibitors ever found, and a couple of TSAs are starting now in clinical trials [9]. Striking cases fuse the TSA inhibitors of purine nucleoside phosphorylase (PNP). PNP is a homotrimeric catalyst that catalyzes the reversible phosphorolysis of 6-oxopurine nucleosides and deoxynucleosides to the relating purine base and a-d-(deoxy) ribose 1-phosphate [10]. PNP is required for boosting the levels of d-guanosine in the blood, realizing apoptosis of confining T-cells in view of the collection of deoxyguanosine triphosphate, an inhibitor of ribonucleotide reductase. Inhibitors of PNP can be used to treat T-cell tumors and insusceptible framework diseases including gout, rheumatoid joint torment, psoriasis, tissue transplant release, and various scleroses. TSA inhibitors of PNP consolidate immucillin-H (now in Stage II clinical trials under the name of forodesine) and DADMe-immucillin-H (passed Stage IIb clinical trial under the name of ulodesine), which were found by Ho et al. [11]. The same group did amazingly cautious test examinations to depict the auxiliary determinants of the affiliation and what is more the energy of PNP impediment by TSA ligands. Besides, few precious stone structures have been determined for PNP, both in the apo and holo structures, thereby giving a considerable measure of data related to TSA ligands in complex with this protein. In any case, the system of a TSA inhibitor official to PNP has not so far been clarified at the atomistic level. From the computational position, PNP is a trying and by and large gigantic system (around 100,000 particles, including dissolvable). Basically, a couple of dynamic rate constants related to intense PNP-TSA inhibitors are at present open. In that limit, we considered PNP-TSA buildings to be perfect demonstrating justification for as of recent advancements in computational tools and hypotheses. In this work, we consolidate microsecond-long MD simulations with machine learning calculations to perceive the basic and dynamical components of a TSA inhibitor authoritative to PNP. We realize a fuzzy-ART methodology [12] to extricate an arrangement in the form of human-interpretable and imperative mesostates from lengthy MD bearings.

The convention is totally automated, as the amount of groups is naturally distinguished and requires no inter-mediation. In purpose of interest, we look into the coupling system of DADMe-immucillin-H to the PNP chemical, in like manner giving the free vitality profiles along the coupling course [13]. DADME compares to a tight coupling TSA inhibitor of PNP, exhibiting an impediment predictable (Ki) of $9 \mathrm{PM}$ and time (the opposite of the koff) of $20 \mathrm{~min}$. We exhibit that our convention can perceive coupling pathways for DADME official to PNP and to depict each one of the courses from the robotic and vivacious perspectives. Inquisitively, DADME official to PNP shows a startling restricting way and extremely unique components, including a moderate-onset deterrent [14]. The coupling procedure recreated here is in full simultaneousness with the available trial revelations [15]. The bound state, as obtained through microsecond time lapse of MD simulations, is amazingly similar to the crystallographic structure of PNP in complex with DADME (numbering structures having root mean square deviation [RMSD] $0.6 \AA \AA$ concerning the precious stone). Besides, we get an evaluation of the coupling kon, which is in simultaneousness with test data. Scaled MD [16] is used to examine the unbinding system and delineate unpretentious components related to the DADME-PNP partition process. In light of this dynamical picture, we drive a novel hypothesis of the moderate-onset tight restricting obstruction component of DADME toward PNP. 


\section{METHODOLOGY}

\section{MD simulations}

The SIESTA system is used for simulations fusing of molecular kinetic data and uneven MD, $a b$ initio quanto-mechanical calculations for ligand and phosphate charge parameterization. The PNP homotrimeric unit was reproduced in conditions of full phosphate inundation. The structure was equilibrated in five phases: The first was done in the NVT clusters (i.e., predictable number of particles, volume, and temperature) for $150 \mathrm{ps}$, with each and every atomistic particle constrained, beside those having a spot with waters and ligands, using a symphonious enduring of $40 \mathrm{kcal} / \mathrm{mol} \AA^{-2}$. A second NVT time of 50 ps with obliged spine (consonant reliable of $20 \mathrm{kcal} / \mathrm{mol}^{-2}$ ) was trialed by a third NVT time of 50 ps again with constrained spine, however with a symphonious basic of $10 \mathrm{kcal} / \mathrm{mol} \AA^{2}$. The fourth NVT stage continued running $50 \mathrm{ps}$ with constrained spine and a consonant prerequisite of $1 \mathrm{kcal} / \mathrm{mol} \AA^{-2}$. The last stage included $350 \mathrm{ps}$ in the NPT bunch (i.e., unfaltering number of particles, volume, and temperature) and a target weight of 1 bar. For the creation run, we used ACEMD25 with 2 fs time step, trading partition of $7.5 \AA$, cutoff of $9 \AA$, electrostatic responsibility surveyed every 2 phases, particle mesh Ewald with $1 \AA$ scattering, NVT gathering, and Langevin indoor controller with $300 \mathrm{~K}$ as target temperature and damping of $0.1 / p s$. The last box had a size of $11,086,108 \AA^{3}$ and contained just about 125,000 particles. For each of the 18 duplicates, the ligand positions were subjectively presented outside the protein (similarly for the five acyclovir proliferations). Once the essential proliferation was equilibrated, the others were gained by playing out a controlled MD with NAMD and PLUMED of the equilibrated structure, intending to target initial positions of the ligands.

\section{Machine learning}

The machine learning association in this work is 2 fold to recognize coupling pathways and intermediates, containing in the supplement coding convention and in the non-direct assurance of free vitality estimation. For this, we joined a fuzzy-ART computation with dynamic Boolean network-based classification of clusters [16]. This methodology identifies the point of interest: It clearly minimizes hefty process which has a sensible geometric significance, it passes on particularly the centroids as tests having a spot with the dataset and, finally, it uses an effective fusion system in a multiple data plain that pushes the calculation toward incredible adjacent minima. The RMSD of the staggering number of particles having a spot either with the coupling site or to the ligand was used as group metric. The viability of the instatement strategy was bolstered by the reproducibility of the cluster results, which were autonomous of the requested frame. As a particular note, the code was enhanced and parallelized by the method for multithreading. For the free vitality estimation task, we used regularized least squares computation using a Gaussian bit to nonlinearly embed the mean constrain. Keeping in mind the end goal to ensure numerical security of the course of action of the related straight plan of conditions, the estimation of sigma for Gaussians was principally set to be 29 while guaranteeing that Tikhonov regularization coefficient of $1 \mathrm{e}^{-8}$ was used. These settings allowed getting a smooth, yet in the meantime fitting to data, free vitality twist.

\section{RESULTS AND DISCUSSION}

In Fig. 1, we show the coupling arrangement as procured by gathering each one of the bearings that were delivered on $13 \mathrm{~ms}$ of MD simulations. Each cluster was named by consistence of its medoid. To get the coupling ways, we ran the Dijkstra's most constrained route computation on the batch diagram using edge of weights as the negative logarithm of the amount of moves. The starting center was named "out," while the conclusion center points were named troupes. By then, we iteratively prepared the briefest way and removed the center points until the goal center point got the chance to be blocked off. A couple of calculations can be used to find the most constrained hazardous route in a diagram. The method here used shows up, regardless, some profitable elements, which are here condensed to smaller size. The possible ways for polymerization is consequently perceived with the help of presented method and the route ID as shown in Fig. 1 is ascertain with incredible degree of connectedness. Besides, picks ways do not have any center point (i.e., group) in similar way and thus can be considered as "free." Two particular restricting courses were obtained and named as out bounded, frontal, and gated as shown in figure 1 Upper and frontal courses were characteristic and extremely similar: In both cases, the helix going up against the coupling site fairly lost its wrinkle and allowed the ligand to enter the coupling site either from over the phosphate or from a "frontal" entry, arranged at the interface between two monomers. The third restricting way (gating) was to some degree sudden: The ligand experienced an opening between the helix and the circle facing the coupling site. This segment did not by and large require the helix to lose its pleat. The gating course incited the last restricting setup state, where a root mean

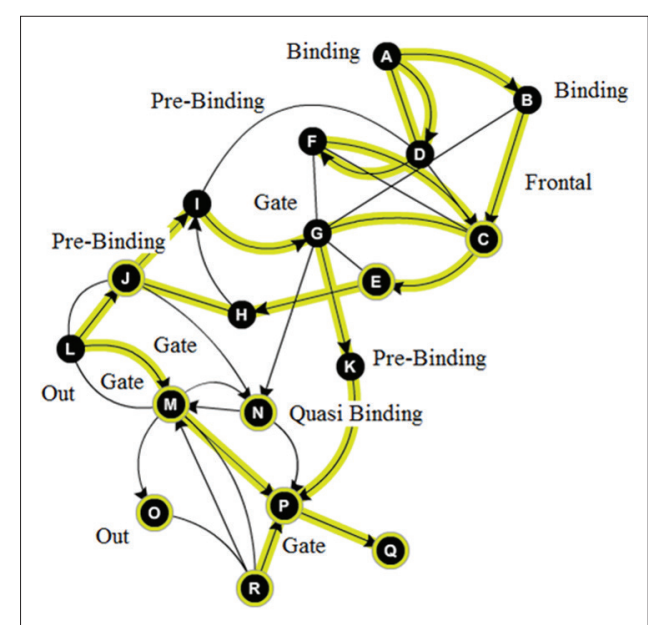

Fig. 1: Clustering of the considerable number of directions by means of fuzzy-ART calculation. Circle highlights encode the group sizes. On every edge, the quantity of moves between associated groups is accounted for. Legends give an engineered depiction of the state and the numbers between props demonstrate the copy to which the medoid of the relating branch begins. The hues encode the most limited ways recognized as coupling courses, so to atomistically depict the coupling pathways and find essential intermediates, we developed an extraordinary convention

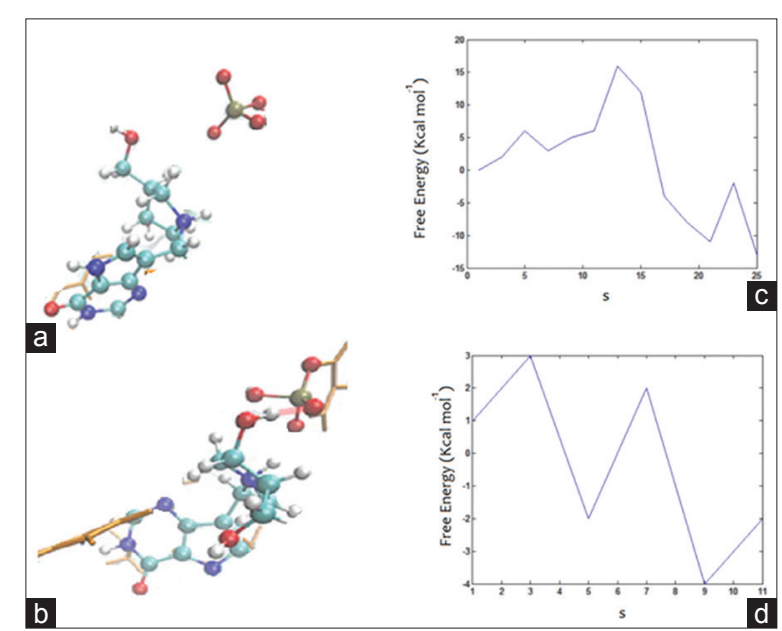

Fig. 2: ( $a$ and $b$ ) the molecular structural arrangement of transitional binding configurations along the two gating mechanisms; whereas the plot (c and d) represents the free energy estimation for the two different binding paths as inferred from fuzzy-ART (a-c) and (b-d). 
square deviation of $0.59 \AA$ versus the crystallographic structure was viewed. By virtue of the upper path, the ligand entered the coupling site exactly when the a-helix adequately left space and the site was revealed. A mandatory conformational alteration of Val260 allowed DADME to accomplish the last restricting game plan, contrasting with outfit A. On official, Val260 recovered the crystallographic consistence.

Free vitality estimation along the coupling pathways is shown in Fig. 2. We then depicted the coupling routes similarly as free kinetic energy. To this point, we performed analyses on simulations along the coupling courses using the way total variable (S-variable) as reaction bearing. The free vitality was then reproduced using a fuzzyART, in Fig. 2c and d, the free vitality profiles along the three ways (gating, frontal, and upper) are represented. The gating component exhibited two minima before accomplishing the last restricting game plan, which identifies with gathering A. These two minima were illustrative of DADME in the portal coordinating toward the dissolvable and toward the coupling site, independently (Fig. 2a and b) for an auxiliary representation of these moderate states). The last state was identified with the crystallographic arrangement, and the free vitality qualification between the fundamental and the last (the gem stance) states was assessed to associate with $13 \mathrm{kcal} / \mathrm{mol}$, in simultaneousness with the test. Strikingly, the impediment for breaking the huge $\mathrm{H}$-bond framework developed among DADME and the developments of PNP reactant site wound up being around $10 \mathrm{kcal} / \mathrm{mol}$, as reported about experimental trial by Kicska et al. on removal of the bi-dentate affiliation. Fig. $2 d$ shows the free vitality along the frontal route from the dissolvable into the reactant pocket going to the crystallographic peak (i.e., troupe A). The free vitality contrast between the bound and unbound states was also for this circumstance around $14 \mathrm{kcal} /$ mol. The likeness of the coupling free vitality contrasts obtained along the two unmistakable courses, in simultaneousness with what must be ordinary from a state limit, substantiated the authenticity of our technique. By virtue of the frontal way, the profile of free energy showed less widely appealing minima differentiated and that of free energy associated with the gating instrument. This was likely due to the nonattendance of complex conformational enhancements like those in the gating system of authoritative. This result was in a conventional simultaneousness with the weak cooperation DADME developed with PNP and with the snappy unbinding component we recognized running scaled MD simulations.

\section{CONCLUSION}

In this study, we have shown that the MD between mechanism of binding between ligand-protein pair can be simulated and described by a genuinely perplexing interchange of intermediary transitioning sets, incorporating differing binding paths and in excess of only a solitary binding arrangement. This method comprehensively covers the dynamic aspects of the process of intermolecular binding. For the moment, regardless of the constraints of the current state of the computational methodologies, we trust that recreation can be a helpful reciprocal instrument to be utilized as a part of collaboration with trials. For instance, we demonstrate that the deliberate utilization of plain and one-sided MD strategies and fuzzy-ART can catch applicable parts of the mechanisms of biomolecular activity recognition and interplay of its interaction.

\section{REFERENCES}

1. Durrant J, McCammon JA. Molecular dynamics simulations and drug discovery. BMC Biol 2011;9:71.

2. Copeland RA, Pompliano DL, Meek TD. Drug-target residence time and its implications for lead optimization. Nat Rev Drug Discov 2006;5:730-9.

3. Jorgensen WL. Foundations of biomolecular modeling. Cell 2013;155:1199-202.

4. Shan Y, Kim ET, Eastwood MP, Dror RO, Seeliger MA, Shaw DE. How does a drug molecule find its target binding site? J Am Chem Soc 2011;133:9181-3.

5. Dror RO, Pan AC, Arlow DH, Borhani DW, Maragakis P, Shan Y, et al. Pathway and mechanism of drug binding to G-protein-coupled receptors. Proc Natl Acad Sci U S A 2011;108(32):13118-23.

6. Pérez-Hernández G, Paul F, Giorgino T, De Fabritiis G, Noé F. Identification of slow molecular order parameters for Markov model construction. J Chem Phys 2013;139(1):015102.

7. Bisignano P, Doerr S, Harvey MJ, Favia AD, Cavalli A, De Fabritiis G. Kinetic characterization of fragment binding in AmpC B-lactamase by high-throughput molecular simulations. J Chem Inf Model 2014;54(2):362-6.

8. Buch I, Giorgino T, De Fabritiis G. Complete reconstruction of an enzymeinhibitor binding process by molecular dynamics simulations. Proc Natl Acad Sci USA 2011;108:10184-9.

9. Rinaldo-Matthis A, Murkin AS, Ramagopal UA, Clinch K, Mee SP, Evans GB, et al. L-Enantiomers of transition state analogue inhibitors bound to human purine nucleoside phosphorylase. J Am Chem Soc 2007;130(3):842-4.

10. Hirschi JS, Arora K, Brooks CL $3^{\text {rd }}$, Schramm VL. Conformational dynamics in human purine nucleoside phosphorylase with reactants and transition-state analogues. J Phys Chem B 2010;114(49):16263-72.

11. Ho MC, Shi W, Rinaldo-Matthis A, Tyler PC, Evans GB, Clinch K, et al. Four generations of transition-state analogues for human purine nucleoside phosphorylase. Proc Natl Acad Sci U S A 2010;107(11):4805-12.

12. Carpenter GA, Grossberg S, Rosen DB. Fuzzy ART: Fast stable learning and categorization of analog patterns by an adaptive resonance system. Neural Netw 1991;4(6):759-71.

13. Poggio T, Girosi F. Networks for approximation and learning. Proc IEEE 1990;78(9):1481-97.

14. Miles RW, Tyler PC, Furneaux RH, Bagdassarian CK, Schramm VL. One-third-the-sites transition-state inhibitors for purine nucleoside phosphorylase. Biochemistry 1998;37(24):8615-21.

15. Lewandowicz A, Tyler PC, Evans GB, Furneaux RH, Schramm VL. Achieving the ultimate physiological goal in transition state analogue inhibitors for purine nucleoside phosphorylase. J Biol Chem 2003;278(34):31465-8

16. Rai A, Ramanathan S, Kannan RJ. Quasi Opportunistic Supercomputing for Geospatial Socially Networked Mobile Devices, 2016 IEEE $25^{\text {th }}$ International Conference on Enabling Technologies: Infrastructure for Collaborative Enterprises (WETICE), Paris; 2016. p. 257-8. DOI: 10.1109/WETICE.2016.65. 